\title{
Ein Fall von tuberculöser Chorioiditis mit spontaner Perforation der Sclera in das Subconjunctivalgewebe.
}

\author{
Von \\ Dr. J. Reissmann in Göttingen.
}

Es sind in den letzten Jahren, nachdem Perls*) den ersten, wenngleich nicht ganz unangefochten dastehenden**) Fall von Iristuberculose veröffentlicht hat, mehrfache Beobachtungen über tuberculöse Erkrankungen einzelner Gebilde des Auges, so der Conjunctiva und der Iris, beschrieben worden.

Bezüglich der hier einschlägigen, genaueren Litteraturangaben verweise ich auf die Arbeiten von Hänsell ***) „Beiträge zur Lehre von der Tuberculose der Iris, der Cornea und Conjunctiva nach Impfversuchen an Thieren und klinischen Beobachtungen an Menschen", von O. Haab $\dagger$ ) „Die Trberenlose des Auges", sowie auf diejenigen von

*) v. Graefe's Archiv f. Ophthalm. Bd. XIX., Abtheilung 1, pag. 221-248. p. 199.

**) cfr. Baumgarten, Archiv f. Ophthalmol., Bd. XXIV. 3,

***) v. Graefe's Archiv f. Ophthalm, Bd. XXV. 4, p. 1.

f) Dasselbe. Bd. XXV. 4, p. 163. 
A. Brückner*) und A. Costa-Pruneda. ${ }^{* *}$ ) - Durch die Resultate der in jenen Publicationen mitgetheilten Beobachtungen ist die bereits früher bekannt gegebene, später jedoch wieder stark angezweifelte Thatsache von neuem bewiesen, dass es ausser den eitrigen Entzündungen der oben erwähnten Augenmedien auch solche von specifisch tubereulösem Charakter giebt, mit den, der Tuberculose allein zukommenden charakteristischen, knötchenartigen Erhebungen, so dass die im klinischen Bild als gelbe Glaskörperinfiltration sioh darbietende Erscheinung als eine wirkliche tuberculöse Production und nicht als eingelagerte Käsemassen aufzufassen sind.

Ein Fall, welcher das Vorkommen derartiger tuberculöser Entzündungen von neuem auf das prägnanteste bestätigt, ist in der Göttinger Universitäts-Augenklinik im Laufe des Sommersemesters 1883 vorgekommen und mir durch die Güte des Herrn Professor Leber zur Untersuchung und Veröffentlichung überlassen worden. Die Publication desselben dürfte vielleicht deshalb von besonderem Interesse sein, weil die Differentialdiagnose zwischen einer durch die, in der Anamnese erwähnten, vielfachen, eitrigen Hautulcerationen hervorgerufenen metastatischen Chorioiditis suppurativa und einer tuberculösen Entzündung der Aderhaut, ausser durch den pathologischanatomischen Befund, und abgesehen von der taberculösen Natur der Hautaffectionen, durch die für das klinische Bild der tuberculösen Entzündung charakteristische, langsamere Entwickelung der Affection in hervortretender Weise bestätigt wurde (cfr. Anamnese). Ausserdem ist der Fall mit Hilfe der neuesten Errungenschaften auf dem Gebiete der Tuberculose, nämlich der Bacillen-Darstellung, untersucht worden, sodass die klinische Diagnose

*) v. Graefe's Arch. f. Ophthalm. Bd. XXVI. 3, p. 154.

**) Dasselbe. Bd. XXVI. 3, p. 174. 
anch auf diese Weise, neben erfolgreieher Ueberimpfung auf das lebende Kaninchen, bestätigt werden konnte.

Ich entnehme den klinischen Journalen der Göttinger Universitäts-A ugenklinik folgenden Krankheitsbericht:

„Am 19. Mai 1883 wurde die 49 Jahre alte Frau Marie Heringlake aus Rischenau in die Klinik aufgenommen. Die Anamnese ergab folgendes: „Patientin giebt an, als Kind stets gesund gewesen zu sein. Von den Eltern soll der Vater an einem Magenleiden (er habe in den letzten Tagen seines Lebens keinerlei Speisen oder Getränke mehr hinunterbringen können) (Carcinoma Oesophagi?) gestorben, die Mutter einer Lungenentzündung erlegen sein. Von 12 Geschwistern sind mehrere ganz gesund, eine Schwester soll im siebenten Lebensjahre ,an den Drüsen" gestorben, eine andere seit 3 Jahren in Folge von Rückenmarksschwindsucht bettlägerig sein. Patientin selbst will vor längeren Jahren an Durchfällen, vor nicht so langer Zeit einmal an „Brustkrankheit” gelitten haben. Die Lungen seien nach Angabe des Arztes nicht ganz gesund gewesen, doch hat Patientin weder am Blnthusten gelitten, noch ist sie bettlägerig gewesen, sie will viel grauen Schleim ausgeworfen haben. - Den Beginn der Augenaffection will Patientin ,vor sechs Wochen" verspürt haben. Auf meine Bitte, mir über den Anfang ihres Leidens noch nähere Angaben zukommen zu lassen, soweit sie sich noch darauf zu besinnen vermöge, theilte mir die Kranke in einem Briefe vom 19. October 1883 dem Inhalte nach ungefähr folgendes mit:

„Vor etwa $2^{1 / 2}$ Jahren bekam ich am rechten Arm fünf kleine Geschwüre, welche nach kurzer Zeit durchbrachen, der Arm war dabei etwas angeschwollen und sah fenerroth aus. Im Februar d. J. verspürte ich an der dem kranken Auge entsprechenden Seite unaufhörliche Schmerzen in der Stirngegend, dann im Hinterkopfe, zuletzt auch in der ganzen rechten Gesichtshälfte, welche 
dabei leicht geschwollen war. Etwa vier Wochen später bekam ich ein juckendes Gefühl am rechten Auge, welches leicht mit Blut unterlaufen war. Abends in der Dämmerung war es mir oft, ,als sähe ich einen zuckenden Blitz, wovon ich den Tag über nichts bemerkte. Dann *) entstand auf dem rechten Ange ein Geschwür, in Folge wovon sich nach und nach das Sehvermogen verlor."

Die objective Untersuchung ergab folgendes Resultat: „Am rechten Auge grosser subconjunctivaler Abscess mit Perforation der Solera. Iritis. Eitrige Chorioiditis vermuthlich tuberculoser Natur. Absolute Amaurose.

Ueber der linken Lunge unten ist etwas gedämpft tympanitischer Schall, das Athmungsgeräusch ist an der betreffenden Stelle abgeschwächt. Catarrh der linken Spitze. An der rechten Lunge ist nichts Abnormes nachzuweisen. Patientin hat schlechten Appetit, eine stark belegte Zunge, sehr cariose Zähne und in Folge dessen Ulcerationsprocesse am Zahnfleischrande.

An den oberen Extremitäten, besonders rechts, finden sich zahlreiche tuberculöse Hautgeschwüre. Dieselben bestehen angeblich seit zwei Jahren und sind alle aus weichen, nach und nach sich entrickelnden Knoten, die nicht schmerzhaft waren, entstanden. Letztere offneten sich zuletzt schon spontan und entleerten Eiter. Die Narben sind bläulich-roth und mit dünner Epidermis bedeckt, unter ihnen ist die Haut infiltrirt.

An der rechten oberen Extremität findet sich eine Ulceration von länglicher Gestalt am unteren Rande der Sehne des Latissimus dorsi, mit centraler Fistel. Zwei weitere, bis auf das Centrum, das unit schlaffen, nicht stark wuchernden Granulationen angefüllt ist, vernarbte Geschwüre zeigen sich an der medialen Seite des Ober-

*) Vermuthich einige Wochen vor der ersten Vorstellung: in der Augenklinik (cfr. die Zeitangabe der Patientin am Schluss der Anamnese). 
armes dicht unter der Achselhöhle. Zwei andere bereits vernarbte, von länglicher Form, liegen medianwärts oberhalb des Ellenbogengelenkes.

Auf der Mitte des Vorderarmes, und zwar an der Extensorenseite, findet sich eine nicht selr umfangreiche, noch leicht ulcerirte, unregelmässig begrenzte Fläche. Fin grösstentheils vernarbtes Geschwür ist ferner sichtbar auf der Volarseite des Unterarms, an der Grenze des oberen Drittels gegen das mittlere. Am Radialrande der Volarseite des Vorderarmes, dicht oberhalb des Handgelenks, sitzt ein vor Kurzem aufgebrochener, kirsehgrosser, schlaffer Knoten.

An der Hand finden sich rechterseits vernarbte Geschwüre zwischen Daumen und Zeigefinger am Rande der Haut, flache Narben auf dem Metakarpalknochen und auf der Dorsalseite des Daumens.

An der linken oberen Extremität sieht man ein Geschwür an der medialen Seite des Oberarms, in der Nähe der Achselhöhle, und zwei weitere vernarbte auf der Beugeseite der Oberarmmitte.

Ein geringer Rest von Ulcerationen ist noch sichtbar an der ersten Phalanx des Daumens der linken Hand. Die Endphalange des Mittelfingers ist kolbig verdickt und zeigt eine Fistel, die unter das Nagelbett fübrt. Nach Angabe der Patientin ist an dieser Stelle vor etwa drei Wochen ein Sequester extrahirt worden."

Ueber die oben beschriebenen Hautgeschwüre äussert sich Herr Professor Rosenbach, dem die Kranke zur Untersuchung vorgestellt wurde, in einer Notiz vom 30. Mai, welche ich mit gütiger Erlaubniss des Herrn Professors hier einfuge, wie folgt:

„An manchen der Ulcerationen konnte man zweifeln, ob man an alte Lues denken solle. Die meisten aber haben einen so ausgesprochenen tuberculösen, und zwar rein 
tuberculösen Charakter, dass nach meiner Ansicht die Diagnose auf Tuberculose unzweifelhaft ist."

Am 21. Mai wurde der kranke Bulbus durch Herrn Professor Leber enucleirt. In dem betreffenden Journal finde ich über die Operation selbst Folgendes verzeichnet:

„Der nach aussen unten sitzende subconjunctivale Abscess, welcher an einer kleinen Stelle die Conjunctiva schon $\mathrm{zu}$ durchbrechen beginnt, wird nach vorausgegangener Tenotomie des Rectus internus, sup. und inf., umschnitten, wobei sich das umgebende Gewebe stark verdickt zeigt. Plötzlich entleert sich, ohne dass der Bulbus angeschnitten ist, eine ziemliche Menge gelblicher, seröser Flussigkeit, wobei der Bulbus collabirt. Die Tenotomie des Externus lässt sich trotzdem glatt ansführen und die Operation normal zu Ende bringen. Es entleert sich dabei nur wenig eitrige Substanz, da dieselbe eine compactere, derb gallertartige Consistenz besitzt."

„Section des Bulbus: Bulbus durch die Mitte des Abscesses meridional eröffnet. Es zeigt sich dabei eine ausgedebnte ulceröse Perforation des Bulbus mit Zerstörung der Sclera und Chorioidea nach aussen unten, dicht hinter der Ora serrata bis zum Aequator hin. Weiter nach vorn ist die eitrige Masse unter der Conjunctiva infiltrirt. Die Netzhaut ist nicht abgelöst. Die Innenfläche der Aderhaut ist von zahlreichen, gelben Knötchen bedeckt, deren Zahl und Grösse nach vorn hin zunimmt, und welche deutlich durch die Retina durchschimmern. Die Retina, leicht verdickt, zeigt auf der Papille und auch sonst einige kleine Extravasate und einzelne, feine, gelbliche Punktchen, zweifelhaft, ob Tuberkeln oder Fettdegenerationsherde. Die Zonula und der vorderste Theil der Netzhaut stärker verdickt, besonders in der Umgebung des Abscesses undurchsichtig, erstere zum Theil von fast knorpeliger Consistenz. Glaskörper verdichtet und von sehr zarten, diffusen Trubbungen durchsetzt. In der Umgebung der Perforations- 
stelle ist die Sclera verdünnt und erweicht. Die Linse ist durchsichtig. Der Ciliarkörper bietet von innen nichts auffalliges." (Am 1. Juni ist die Patientin als geheilt entlassen.)

Die Bulbushälften, welche anfangs 14 Tage lang in verdünntem, etwa 40 procentigen Alcohol gelegen hatten, wurden in absoluten Alcohol gebracht, sodann in Celloidin eingebettet und mit dem Mikrotom geschnitten. Es stellte sich dabei heraus, dass an einer Stelle, etwas nach aussen und unten, dicht hinter der Ora serrata, Chorioidea, Netzhaut und Glaskörper einerseits, und Selera mit subconjunctivalem Gewebe andererseits zu einer gelatinösen Masse verschmolzen, resp. ein grösserer Aderhautherd nach aussen und innen perforirt war and die umgebenden Augenhäute in die Degeneration mit hineingezogen hatte. Der Hauptherd resp. die tumorartige Infiltration des Bulbus hatte sich nach aussen unten in und auf der Selera entwickelt und so den bei der Operation angeschnittenen, scheinbaren Abscess vorgetäuscht.

Die specielle mikroskopische Untersuchung zeigt nun, dass dieser tumorartige Theil in und auf der Selera aus einer dichten Zelleninfiltration besteht, zwischen welcher theilweise ganz unversehrtes Scleralgewebe erkennbar bleibt, theilweise dieses gänzlich in der zelligen Masse aufgegangen ist, so dass nur hier und da noch Reste der Scleralfasern als aufgesplitterte Bindegewebsfäden sichtbar sind. Die Zellen, welche die Masse des Tumors zusammensetzen, sind zum grossen Theil Rundzellen, zum anderen aber grössere epithelioide, sowie vereinzelte Riesenzellen. Nur an wenigen Stellen bietet sich noch ein Bild dar, wie es einem miliaren Tuberkel zukommt, nämlich um eine centrale Riesenzelle oder statt deren eine gelblich-bröcklige, structurlose Masse sind erst epithelioide Zellen, weiter peripher aber Rundzellen so gelagert, dass das ganze Gebilde einen rundlichen Herd vorจ. Graefe's Archiv für Ophthalmologie, XXX. 3. 
stellt. Sonst herrscht meist das Aussehen einer ganz diffusen Infiltration mit den oben schon beschriebenen Zellformen vor, zwischen denen sich hiex und da Fettkörnchenzellen, $a b$ und zu etwas versprengtes Pigment, oder aber eine grössere Menge gelber, bröckelig zerfallener, structurloser Masse vorfindet. Capillargefässe sind, wenngleich spärlich, hier und da erkennbar. Mehr gegen den Rand der Hauptinfiltration, d. h. also gegen die periphere Grenze derselben, an der Sclera, werden die Gefässchen zahlreicher. Das Subconjunctivalgewebe oberhalb dieser Scleralveränderung ist gleichfalls mit in diesen Zellenmassen aufgegangen, bis an den Rand der Cornea hin, während sich das Epithel darüber normal verhält.

Nach innen hin communicirt nun diese eben beschriebene Scleral-Infiltration mit einer nahezu gleichen der mit ihr dicht zusammenhängenden Aderhaut.

Das eigentliche Aderhautgewebe ist an dieser einen circumscripten Stelle in einer gleichen Zelleninfiltration zu Grunde gegangen, wie auch die Sclera, nur dass hier der Reichthum an Pigment ein bedeutend grösserer ist. Ausserdem hat sich auch der Charakter der Einzelherde, nämlich centrale Riesenzelle resp. käsige Substanz mit umgebenden Epithelioid- und Rundzellen etwas mehr erhalten als in jener Scleralwucherung. $A b$ und $\mathrm{zu}$ sind einige kleinere Einzelherde durch feinere, mehr oder weniger vollständige Pigmentstreifen von einander geschieden. Die übrige Chorioidea zeigt in ihrer ganzen Fläche, bis zur Ora serrata hin, die schon makroskopisch gesehenen Einzelherde von gelblicher Farbe. Sie bestehen mikroskopisch, und zwar gewőhnlich jeder makroskopische Einzelherd, aus zwei oder drei kleineren rundlichen Gebilden, die durchaus nach dem Typus eines miliaren Tuberkels aufgebaut sind, nämlich auch hier wieder aus centralen Riesenzellen mit deutlich randständigen Kernen, oder einem gleich geformten Gebilde, das mehr glänzend 
und gelblich aussieht, in dem sich nur sehr schlecht Kerne färben lassen, ferner umgebenden Epithelioid- und endlich Rundzellen. Anstatt der centralen Zellgebilde oder deren Resten findet sich auch wohl hier und da nur eine Lücke, aus der wohl die bröckelig-käsige Substanz herausgefallen ist. Getrennt sind die miliaren Einzelherde durch Züge von Pigmentzellen. Gefässe finden sich in den Herden nicht. Die übrige Chorioidea, soweit sie nicht herdweise in der beschriebenen Art verändert ist, zeigt einfache Rundzellen-Infiltration.

Ciliarkörper und Iris sind ebenfalls nur von mehr oder minder zahlreichen Lymphkörperchen durchsetzt, die Iris ausserdem mit ihrem Pupillarrande der vorderen Linsenkapsel circulär adhärirend. Die Verwachsung daselbst ist eine sehr innige, aus einem festen Spindelzellengewebe mit eingestreutem Pigment bestehend.

Cornea und linse sind normal.

Was nun die Retina anlangt, so ist sie an der oben schon beschriebenen Stelle, da, wo sie mit der degenerirten Aderhaut fest verbunden ist, wie Sclera und Aderhaut auch, in dem gleichen degenerativen Process aufgegangen, der deshalb wohl keiner weiteren detaillirten Beschreibung bedarf. Im Uebrigen ist sie theils mit Rundzellen, theils mit Fettkörnchenzellen durchsetzt. Die Stäbchen- und Zapfensehicht ist mehr oder weniger, je nach den darunter befindlichen Aderhauttuberkeln, zerstört.

Die Sehnervenpapille resp. der Opticus selbst gingen leider für die mikroskopische Untersuchung verloren, so dass nur der makroskopische Befund, wie er oben schon erwähnt wurde, nämlich einzelne Extravasate, sowie einzelne feine, gelbe Pünktehen hier erwähnt werden können. Es muss leider ungewiss bleiben, welcher Natur diese gelben Pünktchen gewesen sind. 
Der Glaskörper endlich ist an der Stelle der Hauptentartung, da, wo er mit Netzhaut und Chorioidea untrennbar zusammenhängt, in Bezug auf das mikroskopische Bild von jenen beschriebenen kaum unterschieden. Nur dass sich zwischen den von daher schon bekannten Zellenelementen noch spindelförmige Zellgebilde einschieben mit grossem Kern, hier und da einer Vacuole, sonst feinkörnigem, glänzenden Protoplasma, Zellgebilde, welche den normalen Glaskörperzellen sehr ähnlich sahen, und wie sie sich bei entzündlichen Augenaffectionen ja fast immer in sehr reichlicher Menge im Innern des Glaskörpers vorfinden. Der übrige Glaskörper ist reichlich mit Fibrin durchsetzt, ferner grösseren und kleineren Rundzellengebilden, zahlreichen Fettkörnchenzellen sowie den eben beschriebenen spindelförmigen Glaskörperzellen. Doch kommen auch derartige Zellen mit mehreren Ausläufern vor.

Von den versehiedensten Theilen der degenerirten Augenhäute wurden entsprechend feine Schnitte nach der Ehrlich'schen Methode auf Tuberkelbacillen untersucht. Doch gelang es nur, ganz vereinzelte, nach mehrtägiger Färbung mit Gentianaviolett dunkel blauschwarz tingirte, schlanke Stäbchen aufzufinden, die indessen in so geringer Anzahl nur wahrgenommen werden konnten, dass darauf hin eine absolut sichere Entscheidung, dass es typische Tuberkelbacillen waren, zunächst allein nicht ausgesprochen werden konnte.

Mit gütiger Erlaubniss des Herrn Professor Leber reihe ich hier die von ihm ausgeführten Uebertragungsversuche von tuberculösem Material aus dem Auge der Frau H. auf das lebende Kaninchenange nach den mir übergebenen Versuchsprotocollen an:

Protocoll: "Von dem frisch enucleirten Auge der Patientin entnahm Herr Professor Leber zwei kleine Stückchen aus der Gegend der Scleralperforation und ver- 
impfte sie nach Anlegung eines Lanzenschnittes in die vordere Augenkammer zweier Kaninchen, je in ein Auge dieser Versuchsthiere. Der Versuch verlief bei beiden Thieren anfänglich in der bekannten Weise, d. h. die eingebrachten Impfstückchen wurden nach und nach resorbirt, ohne dass irgend eine Reizerscheinung auftrat als anfänglich etwas Injection, die aber bald wieder zurückging. Bei dem einen Versuchsthiere blieb. das Auge nach vollständiger Resorption des eingeimpften Stückes eine geraume Zeit lang absolut normal. Nach einer Beobachtung ron 55 Tagen war noch keine Tuberkeleruption in dem betreffenden Auge erfolgt. Da die Impfung ohne Resultat geblieben schien, wurde das Thier erst am 11. October 1883 wieder angesehen, und jetzt unerwartet eine allerdings sehr ausgesprochene Impftuberculose bei diesem Kaninchen entdeckt. Die vordere Kammer war nach aussen und unten mit einer k̇äsigen Masse ausgefüllt, und es zeigte sich eine deutliche Durchwucherung der Sclerocornealgrenze mit einer gelblichgrau gefärbten Masse. Bei dem zweiten Thiere wurde ebenfalls eine Zeit lang, drei Wochen, nach der Einimpfung noch keine Spur von Tuberkeleruption constatirt und daher das Thier erst nach weiteren vier Wochen wieder angesehen. Dabei zeigte sich denn eine ganz exquisite Iristuberculose: Grosser, gelber Knoten von Erbsengrösse im lateralen Theile der Iris, sehr zahlreiche miliare Knötchen auf der ganzen Iris zerstreut, nach unten im Bereich der Sclera mehrere grosse, gelbliche, subconjunctivale Buckel. Am Tage nach dieser Untersuchung fand sich das Thier todtgebissen im Stalle. Das erkrankte Auge wurde enucleirt und in Müller'sche Flüssigkeit gelegt. Die Section des Thieres ergab im Uebrigen keine tuberculose Affection der sonstigen Organe.

Die pathologisch-anatomische Untersuchung des zuerst inficirten Kaninchenauges ergab eine typisshe Tuberculose 
der Iris, des Ciliarkörpers, eines Theiles der Cornea, sowie tuberculöse Infiltration der Sclera in der Ciliargegend, d. h. es finden sich typische miliare Herde mit centraler Riesenzelle, umgebenden epithelioiden, schliesslich peripher gelagerten Rundzellen. Zwischendurch vorgeschrittene Verkäsung. Feinere Schnitte, nach der Ehrlich'schen Methode gefärbt, ergaben eine ausserordentliche Menge typischer Tuberkelbacillen.

Ueberblicken wir nun noch einmal kurz die Ergebnisse obiger Untersuchungen, so dürfte wohl schon der pathologisch-anatomische Befund des Auges der Frau H. allein kaum einen Zweifel an der Diagnose einer echten Tuberculose des Auges aufkommen lassen. Neben schon fortgeschrittener Verkäsung fanden wir in den einzelnen Geweben des Auges typische miliare Herde frischeren Datums, die mit centralen Riesenzellen, sowie umgebenden Epithelioid- und Rundzellen durchaus diejenigen pathologisch-anatomischen Anforderungen erfüllen, die wir an eine typische Tuberculose zu stellen gewohnt sind. Unterstützt wird diese Diagnose jedoch noch durch den Befund von Tuberkelbacillen; dass die wenigen, in dem Bulbus der Frau H. gefundenen Stäbchen wirklich als Tuberkelbacillen aufzufassen sind, dafür spricht wohl der Umstand, dass die gleichen Gebilde, nur sehr viel zahlreicher und deutlicher, in dem zuerst mit Erfolg geimpften Kaninchenbulbus gefunden wurden, wo gar kein Zweifel über ihre Natur aufkommen konnte. Wenn dieselben auch in dem enucleirten Bulbus der Frau H. nur sehr vereinzelt vorhanden waren, so lässt sich diese Thatsache daraus vielleicht erklären, dass das Auge, welches sich bereits in einem weit vorgeschrittenen Stadium der Tuberculose befand, nur spärlich Bacillen, aber dafür Sporen enthielt, die sich durch Färbung nicht sichtbar machen lassen. Da ausserdem der oben angeführte makroskopische Befund in beiden Kaninchenaugen sowohl wie das pathologisch- 
anatomische Untersuchungsresultat des zweiten das ganz charakteristische Bild der Tuberculose darbietet, so dürfte auch noch dadurch die Diagnose, dass es sich in dem beschriebenen Krankheitsfalle um eine echte Tuberculose des Auges der Patientin handelt, als vollständig gesichert $\mathrm{zu}$ betrachten sein.

Bemerkenswerth ist noch, dass die auffällig langsame Entwickelung der tuberculösen Entzündung im Auge der Patientin sowohl wie besonders in den beiden Kaninchenaugen in ganz offenbarem Einklang steht zu der spärlichen Bacillenmenge, die sich im Auge der Kranken fand.*) Wahrscheinlich war von den Bacillen eine Anzahl durch die weit vorgerückte Entzündung bereits $\mathrm{zu}$ Grunde gegangen.

Was nun den Ausgangspunkt der tuberculösen Affection in dem Auge der Patientin, Frau H., betrifft, so ist es wohl das wahrscheinlichste, anzunehmen, dass der Anfangsherd der Erkrankung in der Chorioidea gesessen habe. Da die Iris und das Corpus ciliare von Tuberculose frei waren, ebenso die Cornea, auch das Epithel der Conjunctiva über dem Hauptentartungsherde intact, endlich im Corpus vitreum und der Retina mehr secundäre Infiltrate als eine Haupteruption von Tuberkeln war, so könnte man schon per exclusionem nur auf die mit ausserordentlich zahlreichen miliaren Tuberceln besetzte Aderhaut recurriren.

In der That dürfte es also am ungezwungensten sein, anzunehmen, dass die ersten Erkrankungsherde tuberculöser Natur in der Chorioidea, dicht am Aequator Bulbi, nach aussen unten, gesessen haben, von dort nach innen und

*) Wenn auch die Zeit des Beginns der tuberculösen Affection bei den Thierversuchen nicht direct beobachtet wurde, weil die Impfung anfangs resultatlos geblieben zu sein schien, so ist doch sicher, dass am einen Kaninchenauge nach 22 Tagen, am andern nach 55 Tagen noch nichts von Impftuberculose vorhanden war; dieselbe musste am ersten Auge zwischen dem 22. und 55. Tage, am andern zwischen dem 55. und 143. Tage entstanden sein. 
aussen perforirt seien, und so die Retina und Corpus vitreum einerseits inficirt haben, andrerseits zu der tumorartigen Wncherung in der Sclera und dem subconjunctivalen Gewebe geführt haben.

Zum Schluss kann ich nicht umhin, Herrn Professor Leber sowie Herrn Professor Deutschmann für die Anregung zu dieser Arbeit, sowie für die mir za Theil gewordene Unterstützung meinen besten Dank auszusprechen. 